\title{
man \\ Blood Group Type Association with Head and Neck Cancer
}

\author{
Gaube Alexandra ${ }^{1}$, Michire Alexandru ${ }^{2,3, *}$, Calangiu Filip Stefan ${ }^{3}$, Draghia Petruta-Maria ${ }^{3}$, \\ Burlacu Mihnea Gabriel ${ }^{3}$, Georgescu Dragos-Eugen ${ }^{2,4}$ and Georgescu Mihai Teodor ${ }^{2,3}$
}

1 National Institute of Infectious Diseases “Prof. Dr. Matei Bals”, 021105 Bucharest, Romania; gaube_alexandra@yahoo.com

2 Department 8 Radiology, Oncology, Hematology, "Carol Davila” University of Medicine and Pharmacy, Bulevardul Eroii Sanitari 8, 050474 Bucharest, Romania; gfdragos@yahoo.com (G.D.-E.); georgescumihaiteodor@gmail.com (G.M.T.)

3 Radiation Therapy Department, "Prof. Dr. Al. Trestioreanu" Oncology Institute, Sos. Fundeni No. 252, 022328 Bucharest, Romania; calangiufilip@yahoo.com (C.F.S.); dpetruta@gmail.com (D.P.-M.); mihnea.burlacu93@gmail.com (B.M.G.)

4 Clinical Hospital "Dr Ion Cantacuzino", 030167 Bucharest, Romania

* Correspondence: michirealexandru@gmail.com; Tel.: +40-733345948

Citation: Alexandra, G.; Alexandru, M.; Stefan, C.F.; Petruta-Maria, D.; Gabriel, B.M.; Dragos-Eugen, G.; Teodor, G.M. Blood Group Type Association with Head and Neck Cancer. Hematol. Rep. 2022, 14, 24-30. https://doi.org/10.3390/ hematolrep14010005

Academic Editor: Claudio Cerchione

Received: 31 January 2022

Accepted: 28 February 2022

Published: 2 March 2022

Publisher's Note: MDPI stays neutral with regard to jurisdictional claims in published maps and institutional affiliations.

Copyright: (C) 2022 by the authors. Licensee MDPI, Basel, Switzerland. This article is an open access article distributed under the terms and conditions of the Creative Commons Attribution (CC BY) license (https:// creativecommons.org/licenses/by/ $4.0 /)$.

\begin{abstract}
Background: We conducted an analysis to check whether the ABO blood group impacts the susceptibility or protection against different types of head and neck cancers. Method: We analyzed the medical records of 61,899 cancer patients from "Prof. Dr. Alexandru Trestioreanu" Institute of Oncology from Bucharest, along with the corresponding blood group type. Data were scraped using Python. For analysis, we used Chi-square test. Results: The blood group count was A $(245,45.12 \%)$ followed by $0(160,24.66 \%)$, B $(110,20.26 \%)$, and AB $(28,5.16 \%)$. Hypopharyngeal cancer was associated with B group, oral cavity cancer was associated with a lower risk in patients with B group while $\mathrm{AB}$ patients had a higher risk for oral cavity cancer $\left(\chi^{2}=36.136, \mathrm{df}=18, p=0.007\right)$. Conclusion: Blood group B is associated with an increased incidence for hypopharyngeal cancer, whereas, for the oral cavity, was associated lower incidence. Blood antigen A is associated with a higher risk of oral cavity cancer development, independent of B blood antigen.
\end{abstract}

Keywords: blood group; hypopharyngeal cancer; oral cavity cancer; oropharyngeal cancer; protective factor

\section{Introduction}

The ABO blood group systems antigens were discovered in late 1900 and are considered by many the first human genetic markers. [1] Over the last five decades, studies focusing on $\mathrm{ABO}$ antigens found that those are expressed on the surface of many other cells than red blood cells, including epithelial and endothelial cells [2]. This further led to research regarding an existing link between $\mathrm{ABO}$ antigens and chronic diseases, including malignancies [3-5]. It has been recently discovered that the existence of $\mathrm{ABO}$ antigens on the surface of cancer cells, although different from normal cell ABO antigens, is still associated with the ability of these cells to escape an immune system reaction, escaping apoptosis [6].

Recent research in medical oncology focused on different types of cancer, depending on their frequency, identifying a series of diverse factors [7] which influenced the evolution of the disease as well as the treatment outcome. While these results led to new therapeutic approaches [8] which generated better treatment outcomes even in metastatic stages $[9,10]$, cancer is still one of the leading causes of death in the EU and USA [11,12].

Several extensive investigations evaluated the existence of a relationship between the blood group and prognosis for patients diagnosed with malignancies, but even until now, this association remains unclear [13-16]. However, solid results linking the impact of 
the individual's blood group on a certain malignancy risk came from recently published studies such as the one of Yang X. et al. [14] and Kakasava K. et al. [17]

Head and neck squamous cell carcinomas (HNSCC) are the sixth most common human malignancy worldwide, with a continuous rising in incidence up to an estimated $30 \%$ increased incidence in 2030 [18]. High mortality risk factors such as smoking, alcohol consumption and viral infections (e.g., EBV, HPV) are incriminated in the development of HNSCC [19].

Considering previously published data assessing the relative risk of head and neck cancers among different blood groups [20,21], we conducted an analysis to check whether $\mathrm{ABO}$ blood group impacts the susceptibility or protection against different types of head and neck cancers.

\section{Materials and Methods}

We conducted a retrospective analysis on cancer-diagnosed patients admitted at "Prof. Dr. Alexandru Trestioreanu" Institute of Oncology from Bucharest between January 2013 and February 2021. Patients' medical charts were evaluated and besides demographic data (e.g., gender, age), the $\mathrm{ABO}$ blood group and primary site head and neck cancer diagnosis were extracted.

Electronic records were scraped using Python version 3 [22].

Patients were divided in seven head and neck primary malignancy subsites: hypopharynx, larynx, lip, nasopharynx, oral cavity, oropharynx, other, according to ICD-10 coding system [23]. For each group, the existence of a link between primary malignancy diagnosis and blood group was evaluated.

For statistical analysis, we used Chi-square test in R Studio [24] to check whether an association was found between cancer diagnosis and blood group. $p$-values lower than 0.05 were considered as significant. Exploratory analysis was conducted using ggplot2 and mosaic libraries from R Studio.

\section{Results}

We had an initial enrollment of 61,899 patients, and after excluding the patients for which the blood group was missing from their medical charts, 19,626 patients remained. Regarding demographic data, the mean patient age was $64( \pm 22)$ years, whilst the gender stratification resulted in 5773 males and 13,853 females. The most common blood group was A $(44.39 \%)$ followed by $0(33.64 \%)$, B $(17.28 \%)$, and AB $(4.69 \%)$. Selecting for primary head and neck cancer, 543 patients were eligible for analysis.

A total of 121 primary cancer sites were in the oropharynx (6.63\%), 121 in the oral cavity $(22.29 \%)$, and 86 in the larynx $(15.84 \%)$. The most common blood group was A $(245,45.12 \%)$ followed by $0(160,24.66 \%), B(110,20.26 \%)$, and AB $(28,5.16 \%)$ (Table 1$)$.

Table 1. Contingency table for H\&N cancer localization with blood group.

\begin{tabular}{cccccc}
\hline \multirow{2}{*}{ Localization } & \multicolumn{5}{c}{ Blood Type N (\%) } \\
\cline { 2 - 6 } & $\mathbf{O}$ & $\mathbf{A}$ & $\mathbf{A B}$ & $\mathbf{B}$ & $\mathbf{N}(\mathbf{\%})$ \\
\hline Hypopharynx & $6(3.75)$ & $15(6.12)$ & $2(7.14)$ & $13(11.82)$ & $36(6.63)$ \\
Larynx & $22(13.75)$ & $45(18.37)$ & $0(0)$ & $19(17.27)$ & $86(15.84)$ \\
Lip & $4(2.5)$ & $10(4.08)$ & $2(7.14))$ & $9(8.18)$ & $25(4.6)$ \\
Nasopharynx & $27(16.88)$ & $28(11.43)$ & $1(3.57)$ & $12(10.91)$ & $68(12.52)$ \\
Oral cavity & $34(21.25)$ & $61(24.90)$ & $12(42.86)$ & $14(12.73)$ & $121(22.29)$ \\
Oropharynx & $41(25.63)$ & $54(22.04)$ & $9(32.14)$ & $28(25.26)$ & $132(24.31)$ \\
Other & $26(16.25)$ & $32(13.06)$ & $2(7.14)$ & $15(13.64)$ & $75(13.81)$ \\
Total & $160(29.47)$ & $245(45.12)$ & $28(5.16)$ & $110(20.26)$ & $543(100)$ \\
\hline
\end{tabular}

Our study results, as presented in Figure 1, reveal that hypopharyngeal cancer was associated with the $B$ group, oral cavity cancer was associated with a lower risk in patients with $B$ group, while $A B$ patients had a higher risk for oral cavity cancer $\left(\chi^{2}=36.136, d f=18\right.$, $p=0.007)$. 


\section{Mosaic plot for cancer location with blood type}

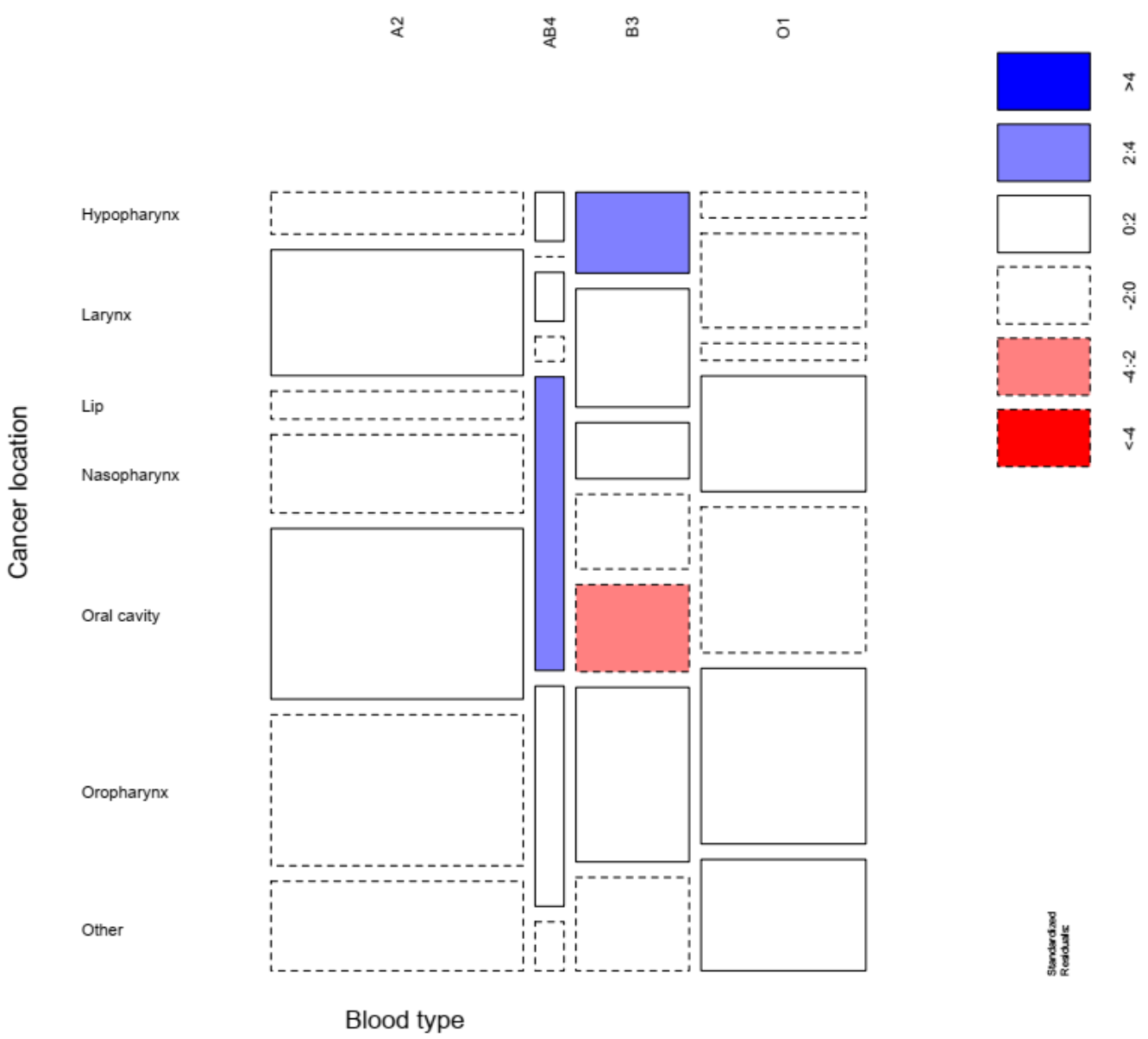

Figure 1. Higher hypopharynx cancer was associated with B group, oral cavity cancer was associated with a lower risk in patients with $B$ group, while $A B$ patients had a higher risk for oral cavity cancer.

\section{Discussion}

Although the first data regarding ABO changes in tumors were reported in 1930, only decades later were changes in the expression of blood group antigens reported in HNSCCs [25]. Regarding the blood group antigens, Hakomori et al. [26] reported that they are mostly expressed in endodermal epithelial cells, coincidence or not, the primary site of most human cancers. Research never ceased to continue in this field, and, more recently, in 2020, a prospective trial [27] on $\mathrm{ABO}(\mathrm{H})$ antigens conducted on 60 patients with oral cavity malignant and potentially malignant lesions, identified that the degree of loss of $\mathrm{ABO}(\mathrm{H})$ antigens in tissue specimens can be used as a marker of tumor stage of the patient. This conclusion resulted from the difference between poorly and moderately differentiated oral cavity carcinomas compared to well-differentiated carcinomas regarding the antigen reactivity.

The studies of Jaleel B. et al. [28] and Singh K. et al. [20] conducted on consistent populations of head and neck cancer patients assessed the relative risk of various HNSCCs amongst different blood groups. After data analysis, both studies were consistent in concluding that precursor $\mathrm{H}$ antigen, a protective factor against oral cancer, transformation to $\mathrm{A}$ and $\mathrm{B}$ antigens increases the risk of developing oral cancer and that $\mathrm{O}$ blood group have the highest $\mathrm{H}$ antigen amount and thus the lowest rates of oral cancer amongst the subjects with this blood group. Two years later, another study's [17] results were published, reporting that on a population of $195 \mathrm{HNSCC}$ patients group A showed the highest potential of developing this malignancy, compared to the other blood groups. Furthermore, in the 
Singh K. et al. [20] study, blood group A was found to be a potential risk factor for the development of salivary gland and esophageal cancer, whereas group B was found to be a potential risk factor for laryngeal cancer.

The association between nasopharyngeal carcinoma (NPC) and ABO blood groups has been previously studied, but the statistical data analysis resulted in conflicting findings. Initially, studies focused on the role of the $\mathrm{ABO}$ blood group as a risk factor for developing NPC. Therefore, while the study of Seow et al. [29] reported no association between ABO blood group and NPC, decades later, Turkoz et al. [30] found that the ABO blood group was related to NPC susceptibility. From the results reported in the latter study, blood type A was related to an increased risk of developing NPC, compared to blood group $\mathrm{O}$, which showed a protective effect. More recent data regarding NPC came from China, where research focused more on the prognostic impact of ABO blood group on NPC. The authors of the initial studies linked the contradictory conclusions of their studies to the area-specific population and to geographic variations worldwide [31-33]. However, two recent studies [31,33] focusing on the population from Southeast China failed to reach a consensus regarding the prognostic value of $\mathrm{ABO}$ blood group on NPC. Moreover, genome-wide association studies [34-36] failed to link ABO blood groups to NPC risk. It is already widely accepted that lymphocyte counts are important prognostic factors for cancer patients [37-40]. The study of Zhang Y et al. [32] is the only one to evaluate the link between the $\mathrm{ABO}$ blood group, tumor stage and blood lymphocyte counts. The authors found that regarding the overall survival (OS) for locally advanced NPC patients with high lymphocyte levels, compared to non-A subgroups, the A blood group patients had better rates. A meta-analysis by Shao-wu Jing showed that, in Chinese patients, blood group $\mathrm{O}$ was associated with a lower incidence of NPC, while group A had no correlation with NPC [41].

The relationship between laryngeal cancer incidence and $\mathrm{ABO}$ blood group was previously studied. Three Polish studies [40,42-44] published between 1992 and 2000 failed to find a link between laryngeal cancer and $\mathrm{ABO}$ blood group. In the studies from Konieczna et al. [42] and Pyd et al. [43], blood group A2 was significantly more frequent in patients with glottic carcinoma, compared to the healthy population or to the other laryngeal subsites. Later on, in 2000, Nowinska et al. [44] failed to find a significant difference among $\mathrm{ABO}$ blood groups between laryngeal cancer patients and healthy individuals. More recently, published studies $[45,46]$ also failed to reach a consensus on the relationship between ABO blood groups and laryngeal cancer. The study of Jin T. [46] et al. is the one with the largest cohort (1477 patients) and which showed that the ABO blood group was an independent prognostic factor for patients with laryngeal carcinoma. Therefore, patients with blood group O diagnosed with laryngeal carcinoma had almost $10 \%$ shorter $3-$-, 5-, and 10 -year OS rates compared to the median OS of the patient population. The limited number of patients enrolled, the lack of strong statistical data, and being single-center studies are the main limitations that explain the current conflicting results for studies evaluating the prognostic value of the $\mathrm{ABO}$ blood group in laryngeal cancers.

The contradictory results regarding the relationship between $\mathrm{ABO}$ blood groups and HN cancers are reported for other sites also. In 2014, a study on 203 differentiated thyroid cancer patients, a lower risk was found for those with blood group B when compared to blood group O [47]. Five years later, another study [48] on thyroid cancer patients reported no significant association between this malignancy and $\mathrm{ABO}$ blood groups. However, in this later study, data analysis resulted in higher rates of extrathyroidal extension and advanced stage disease in B blood groups, compared to their non-B blood group counterparts.

\section{Conclusions}

Alongside the current literature data, our study's results show that different blood groups are associated with different sites where head and neck cancer can arise. In our study, blood groups were found to be an important prognostic factor for tumor development. We identified that $\mathrm{B}$ blood antigen presence acts different according to the tumor site. 
For hypopharyngeal cancer, blood group B is associated with an increased incidence, whereas, for oral cavity, it acts as a protective factor, thus being linked to a lower incidence rate. Additionally, our study found that A blood antigen presence increases the risk of oral cavity cancer development, independent of B blood antigen presence. However, further investigations, including other factors such as environmental, demographic, and behavioral parameters, are still needed in order to corelate blood groups to the susceptibility or protection against different types of cancers.

Author Contributions: Conceptualization, G.A. and G.M.T.; data curation, G.A., M.A. and C.F.S.; formal analysis, M.A.; investigation, M.A., C.F.S. and B.M.G.; methodology, D.P.-M. and G.D.-E.; project administration, G.M.T.; resources, G.D.-E. and G.M.T.; software, M.A. and D.P.-M.; supervision, G.M.T.; visualization, B.M.G.; writing—original draft, G.A.; writing—review and editing, G.A., M.A., D.P.-M., C.F.S., B.M.G., G.D.-E. and G.M.T. All authors have read and agreed to the published version of the manuscript.

Funding: This research received no external funding.

Informed Consent Statement: All patients completed and signed informed consent.

Data Availability Statement: Data is available upon request.

Conflicts of Interest: The authors declare no conflict of interest.

\section{References}

1. Aymard, J.-P. Karl Landsteiner (1868-1943) and the discovery of blood groups. Transfus. Clin. Biol. 2012, 19, 244-248. [CrossRef] [PubMed]

2. Pourazar, A. Red cell antigens: Structure and function. Asian J. Transfus. Sci. 2007, 1, 24-32. [CrossRef]

3. Jesch, U.; Endler, P.C.; Wulkersdorfer, B.; Spranger, H.; ABO blood group. Related investigations and their association with defined pathologies. Sci. World J. 2007, 7, 1151-1154. [CrossRef] [PubMed]

4. Poujol-Robert, A.; Boelle, P.-Y.; Wendum, D.; Poupon, R.; Robert, A. Association Between ABO Blood Group and Fibrosis Severity in Chronic Hepatitis C Infection. Dig. Dis. Sci. 2006, 51, 1633-1636. [CrossRef] [PubMed]

5. Wolpin, B.M.; Chan, A.T.; Hartge, P.; Chanock, S.J.; Kraft, P.; Hunter, D.J.; Giovannucci, E.L.; Fuchs, C.S. ABO blood group and the risk of pancreatic cancer. J. Natl. Cancer Inst. 2009, 101, 424-431. [CrossRef] [PubMed]

6. Zouine, S.; Marnissi, F.; Otmani, N.; Bennani Othmani, M.; Zaid, N.; Kojok, K.; Zaid, Y.; Tahiri Jouti, N.; Habti, N. Expression of Histo-blood Group Antigens in Tumor and Adjacent Normal Breast Tissues as Prognostic Markers of Breast Carcinoma. J. Breast Cancer 2020, 23, 69-79. [CrossRef]

7. Georgescu, D.; Patrascu, T.; Georgescu, T.; Tulin, A.; Mosoia, L.; Bacalbasa, N.; Stiru, O.; Georgescu, M.-T. Diabetes Mellitus as a Prognostic Factor for Locally Advanced Rectal Cancer. In Vivo 2021, 35, 2495-2501. [CrossRef] [PubMed]

8. Moldoveanu, V.G.; Georgescu, M.T.; Dumitrache, M.; Vlad, F.C.; Cioran, N.; Anghel, R.M. Normal brain and organs at risk radiation exposure in multiple coplanar and non-coplanar arc patterns of volumetric-modulated radiotherapy for glioblastoma multiforme. Rom. J. Phys. 2019, 64, 704.

9. Georgescu, M.T.; Moldoveanu, V.; Ileanu, B.-V.; Anghel, R. Dosimetric influence of uterus position in cervix cancer high-dose-rate brachytherapy. Rom. J. Phys. 2016, 61, 1557-1566.

10. Georgescu, M.-T.; Patrascu, T.; Serbanescu, L.; Anghel, R.; Gales, L.; Georgescu, F.; Mitrica, R.; Georgescu, D. When Should We Expect Curative Results of Neoadjuvant Treatment in Locally Advanced Rectal Cancer Patients? Chir. Buchar. Rom. 2021, 116, 16-23. [CrossRef] [PubMed]

11. Ahmad, F.B.; Anderson, R.N. The Leading Causes of Death in the US for 2020. JAMA 2021, 325, 1829-1830. [CrossRef]

12. World Health Organization. The Top 10 Causes of Death. Available online: https://www.who.int/news-room/fact-sheets/ detail/the-top-10-causes-of-death (accessed on 30 January 2022).

13. Liumbruno, G.M.; Franchini, M. Hemostasis, cancer, and ABO blood group: The most recent evidence of association. J. Thromb. Thrombolysis 2014, 38, 160-166. [CrossRef] [PubMed]

14. Yang, X.; Huang, Y.; Feng, J.-F. Is there an association between ABO blood group and overall survival in patients with esophageal squamous cell carcinoma? Int. J. Clin. Exp. Med. 2014, 7, 2214-2218.

15. Qiu, M.-Z.; Zhang, D.-S.; Ruan, D.-Y.; Luo, H.-Y.; Wang, Z.-Q.; Zhou, Z.-W.; Wang, F.-H.; Li, Y.-H.; Xu, R.-H. A relationship between $\mathrm{ABO}$ blood groups and clinicopathologic characteristics of patients with gastric adenocarcinoma in China. Med. Oncol. Northwood Lond. Engl. 2011, 28 (Suppl. S1), S268-S273. [CrossRef] [PubMed]

16. Lee, J.S.; Ro, J.Y.; Sahin, A.A.; Hong, W.K.; Brown, B.W.; Mountain, C.F.; Hittelman, W.N. Expression of blood-group antigen A-a favorable prognostic factor in non-small-cell lung cancer. N. Engl. J. Med. 1991, 324, 1084-1090. [CrossRef] [PubMed] 
17. Kakava, K.; Karelas, I.; Koutrafouris, I.; Damianidis, S.; Stampouloglou, P.; Papadakis, G.; Xenos, A.; Krania, F.; Sarof, P.; Tasopoulos, G.; et al. Relationship between ABO blood groups and head and neck cancer among Greek patients. J. BUON 2016, 21, 594-596. [PubMed]

18. Ferlay, J.; Colombet, M.; Soerjomataram, I.; Mathers, C.; Parkin, D.M.; Piñeros, M.; Znaor, A.; Bray, F. Estimating the global cancer incidence and mortality in 2018: GLOBOCAN sources and methods. Int. J. Cancer 2019, 144, 1941-1953. [CrossRef] [PubMed]

19. Johnson, D.E.; Burtness, B.; Leemans, C.R.; Lui, V.W.Y.; Bauman, J.E.; Grandis, J.R. Head and neck squamous cell carcinoma. Nat. Rev. Dis. Primer 2020, 6, 1-22. [CrossRef]

20. Singh, K.; Kote, S.; Patthi, B.; Singla, A.; Singh, S.; Kundu, H.; Jain, S. Relative Risk of Various Head and Neck Cancers among Different Blood Groups: An Analytical Study. J. Clin. Diagn. Res. JCDR 2014, 8, ZC25-ZC28. [CrossRef]

21. Sharma, G.; Choudhary, R.; Bharti, D. Studies Showing the Relationship between ABO Blood Groups and Major Types of Cancers. Asian J. Exp. Sci. 2007, 21, 4.

22. Van Rossum, G.; Drake, F.L. Python 3 Reference Manual; CreateSpace: Scotts Valley, CA, USA, 2009; ISBN 1-4414-1269-7.

23. World Health Organization. ICD-10: International Statistical Classification of Diseases and Related Health Problems, 10th ed.; World Health Organization: Geneva, Switzerland, 2004.

24. RStudio Team. RStudio: Integrated Development Environment for R; RStudio, PBC: Boston, MA, USA, 2020.

25. Kovarik, S.; Davidsohn, I.; Stejskal, R. ABO antigens in cancer. Detection with the mixed cell agglutination reaction. Arch. Pathol. 1968, 86, 12-21. [PubMed]

26. Hakomori, S. Aberrant glycosylation in tumors and tumor-associated carbohydrate antigens. Adv. Cancer Res. 1989, 52, 257-331. [CrossRef]

27. Pokala, A.; Paramkusam, G.; Tejasvi, M.L.A.; Bangi, B.B.; Nadendla, L.K.; Devulapalli, R.V. Histo-Blood Group Antigens in Oral Cancer and Potentially Malignant Disorders. Asian Pac. J. Cancer Prev. APJCP 2020, 21, 1163-1166. [CrossRef]

28. Jaleel, B.F.; Nagarajappa, R. Relationship between ABO blood groups and oral cancer. Indian J. Dent. Res. 2012, 23, 7-10. [CrossRef]

29. Seow, L.J.; Kwa, S.B.; Teoh, C.K. A preliminary survey of ABO blood group frequency in nasopharyngeal carcinoma in Chinese patients. Singap. Med. J. 1964, 4, 93-95.

30. Turkoz, F.P.; Celenkoglu, G.; Dogu, G.G.; Kalender, M.E.; Coskun, U.; Alkis, N.; Ozkan, M.; Turk, H.M.; Arslan, U.Y. Risk factors of nasopharyngeal carcinoma in Turkey-an epidemiological survey of the Anatolian Society of Medical Oncology. Asian Pac. J. Cancer Prev. APJCP 2011, 12, 3017-3021. [PubMed]

31. Sheng, L.; Sun, X.; Zhang, L.; Su, D. ABO blood group and nasopharyngeal carcinoma risk in a population of Southeast China. Int. J. Cancer 2013, 133, 893-897. [CrossRef]

32. Zhang, Y.-X.; Kang, S.-Y.; Chen, G.; Fang, W.-F.; Wu, X.; You, H.-J.; He, D.-C.; Cao, Y.-L.; Liang, W.-H.; Zhang, L. ABO blood group, Epstein-Barr virus infection and prognosis of patients with non-metastatic nasopharyngeal carcinoma. Asian Pac. J. Cancer Prev. APJCP 2014, 15, 7459-7465. [CrossRef]

33. Lin Lack of Association between the Distribution of ABO Blood Groups and Nasopharyngeal Carcinoma in a Population of Southern China. Available online: https: / / www.cancerjournal.net/article. asp?issn=0973-1482; year=2018; volume=14;issue=4; spage $=785$; epage $=788$; aulast $=$ Lin (accessed on 22 September 2021).

34. Bei, J.-X.; Li, Y.; Jia, W.-H.; Feng, B.-J.; Zhou, G.; Chen, L.-Z.; Feng, Q.-S.; Low, H.-Q.; Zhang, H.; He, F.; et al. A genome-wide association study of nasopharyngeal carcinoma identifies three new susceptibility loci. Nat. Genet. 2010, 42, 599-603. [CrossRef]

35. Ng, C.C.; Yew, P.Y.; Puah, S.M.; Krishnan, G.; Yap, L.F.; Teo, S.H.; Lim, P.V.H.; Govindaraju, S.; Ratnavelu, K.; Sam, C.K.; et al. A genome-wide association study identifies ITGA9 conferring risk of nasopharyngeal carcinoma. J. Hum. Genet. 2009, 54, 392-397. [CrossRef]

36. Tse, K.-P.; Su, W.-H.; Chang, K.-P.; Tsang, N.-M.; Yu, C.-J.; Tang, P.; See, L.-C.; Hsueh, C.; Yang, M.-L.; Hao, S.-P.; et al. Genomewide association study reveals multiple nasopharyngeal carcinoma-associated loci within the HLA region at chromosome $6 \mathrm{p} 21.3$. Am. J. Hum. Genet. 2009, 85, 194-203. [CrossRef] [PubMed]

37. Idos, G.E.; Kwok, J.; Bonthala, N.; Kysh, L.; Gruber, S.B.; Qu, C. The Prognostic Implications of Tumor Infiltrating Lymphocytes in Colorectal Cancer: A Systematic Review and Meta-Analysis. Sci. Rep. 2020, 10, 3360. [CrossRef] [PubMed]

38. Geng, Y.; Shao, Y.; He, W.; Hu, W.; Xu, Y.; Chen, J.; Wu, C.; Jiang, J. Prognostic Role of Tumor-Infiltrating Lymphocytes in Lung Cancer: A Meta-Analysis. Cell. Physiol. Biochem. 2015, 37, 1560-1571. [CrossRef] [PubMed]

39. Alexandru, M.; Rodica, A.; Dragos-Eugen, G.; Mihai-Teodor, G. Assessing the Spleen as an Organ at Risk in Radiation Therapy and Its Relationship With Radiation-Induced Lymphopenia: A Retrospective Study and Literature Review. Adv. Radiat. Oncol. 2021, 6, 100761. [CrossRef]

40. Gao, Z.; Li, C.; Liu, M.; Jiang, J. Predictive and prognostic role of tumour-infiltrating lymphocytes in breast cancer patients with different molecular subtypes: A meta-analysis. BMC Cancer 2020, 20, 1150. [CrossRef]

41. Jing, S.; Xu, Q.; Zhang, X.; Jing, Z.; Zhao, Z.; Zhang, R.; Wu, F.; Wang, J. Are People With Blood Group O More Susceptible to Nasopharyngeal Carcinoma and Have Worse Survival Rates? A Systematic Review and Meta-Analysis. Front. Oncol. 2021, 11, 3258. [CrossRef]

42. Konieczna, A.; Turowski, G. ABO blood groups system in laryngeal cancer patients and after survival of 3 and 5 years. Mater. Med. Pol. 1992, 24, 266-267. [PubMed]

43. Pyd, M.; Rzewnicki, I.; Suwayach, U. ABO blood groups in patients with laryngeal and hypopharyngeal cancer. Otolaryngol. Pol. 1995, 49 (Suppl. S20), 396-398. 
44. Nowińska, E.; Namysłowski, G.; Scierski, W.; Kocierz, S. ABO blood groups in the patients with laryngeal cancer. Otolaryngol. Pol. 2000, 54 (Suppl. S31), 209-211.

45. Adam, S.I.; Wilson, K.M.; Overholser, S.M.; Khabbaz, E.; Moreno, K.; Patil, Y.J. Are laryngeal squamous cell carcinoma incidence and patient mortality a function of ABO blood grouping? A retrospective study. J. Laryngol. Otol. 2012, 126, 180-184. [CrossRef]

46. Jin, T.; Li, P.-J.; Chen, X.-Z.; Hu, W.-H. ABO blood group is a predictor of survival in patients with laryngeal cancer. Chin. J. Cancer 2016, 35, 90. [CrossRef] [PubMed]

47. Lence-Anta, J.J.; Xhaard, C.; Ortiz, R.M.; Kassim, H.; Pereda, C.M.; Turcios, S.; Velasco, M.; Chappe, M.; Infante, I.; Bustillo, M.; et al. Environmental, Lifestyle, and Anthropometric Risk Factors for Differentiated Thyroid Cancer in Cuba: A Case-Control Study. Eur. Thyroid J. 2014, 3, 189-196. [CrossRef] [PubMed]

48. Tam, A.A.; Özdemir, D.; Fakı, S.; Bilginer, M.C.; Ersoy, R.; Çakır, B. ABO Blood Groups, Rh Factor, and Thyroid Cancer Risk: To "B" or Not to "B". Endocr. Res. 2020, 45, 137-146. [CrossRef] [PubMed] 